\title{
Tonic and transient oscillatory brain activity during acute exercise
}

\author{
Luis F. Ciria ${ }^{1,2 *}$, Antonio Luque-Casado ${ }^{1,3}$, Daniel Sanabria ${ }^{1,2}$, Darias Holgado ${ }^{1,4}$, Plamen Ch. \\ Ivanov $^{5,6} \&$ Pandelis Perakakis ${ }^{1,7}$ \\ ${ }^{1}$ Mind, Brain, \& Behavior Research Center, University of Granada, Spain. \\ ${ }^{2}$ Department of Experimental Psychology, University of Granada, Spain. \\ ${ }^{3}$ Faculty of Physical Activity and Sport Sciences, Pablo de Olavide University, Spain \\ ${ }^{4}$ Department of Physical Education \& Sport, University of Granada, Spain. \\ ${ }^{5}$ Keck Laboratory for Network Physiology, Department of Physics, Boston University, USA. \\ ${ }^{6}$ Harvard Medical School and Division of Sleep Medicine, Brigham and Women's Hospital, Boston, \\ USA. \\ ${ }^{7}$ Department of Psychology, Loyola Andalucía University, Spain. \\ *Corresponding author: \\ Luis F. Ciria \\ C/ Campus Universitario la Cartuja, Granada, 18071, Spain. \\ E-mail address: lciria@ugr.es
}

October 11, 2017

\begin{abstract}
The physiological changes that occur in the main body systems and organs during physical exercise are well described in the literature. Despite the key role of brain in processing afferent and efferent information from organ systems to coordinate and optimize their functioning, little is known about how the brain works during exercise. The present study investigated tonic and transient oscillatory brain activity during a single bout of aerobic exercise. Twenty young males (19-32 years old) were recruited for two experimental sessions on separate days. Electroencephalographic (EEG) activity was recorded during a session of cycling at $80 \%$ (moderate-to-high intensity) of $\mathrm{VO}_{2 \max }$ (maximum aerobic capacity) while performing an oddball task where participants had to detect infrequent targets presented among frequent non-targets. This was compared to a (baseline) light intensity session $\left(30 \% \mathrm{VO}_{2 \mathrm{max}}\right)$. The light intensity session was included to control for any potential effect of dual-tasking (i.e., pedaling and performing the oddball task). A warm-up and cool down periods were completed before and after exercise, respectively. A cluster-based nonparametric permutations test showed an increase in power across the entire frequency spectrum during the moderate-to-high intensity exercise, with respect to light intensity. Further, we found that the more salient target lead to lower increase in (stimulus-evoked) theta power in the $80 \% \mathrm{VO}_{2 \max }$ with respect to the light intensity condition. On the contrary, higher decrease alpha and lower beta power was found for standard trials in the moderate-to-high exercise condition than in the light exercise condition. The present study unveils, for the first time, a complex brain activity pattern during acute exercise (at $80 \%$ of maximum aerobic capacity). These findings might help to elucidate the nature of changes that occur in the brain during physical exertion.
\end{abstract}

Key words: Brain function, EEG, fitness, brain rhythms, oddball, exercise intensity, cluster analysis 


\section{Introduction}

The dynamics and regulatory mechanism of body systems and organs such as muscles, joints, heart, lungs, etc. under physical exercise is well described in the literature [1][2][3][4][5][6]. However, little is known about how the brain works when exercising [7][8]. Here, we provide novel evidence on oscillatory brain activity during a single bout of aerobic exercise as a starting point to better understand the highly complex way in which the brain functions during physical exertion.

The study of brain activity in motion entails several methodological and technical issues (e.g. sweating, body movements, muscle potentials, etc.). This is probably the main reason why only a reduced number of studies have investigated brain activity during exercise, primarily using electroencephalography (EEG). The majority of these studies focused on changes in the alpha frequency band at frontal localizations [9] reporting increased alpha activity during exercise [10][11]. The only meta-analysis to date [12], however, indicates that alpha brain rhythm activation is not selective and maybe parallel by an increase of other brain rhythms, suggesting a possible power increase across the entire frequency spectrum during exercise. Notably, these early studies focused on the averaged steady state spectral activation across the entire exercise period and have not investigated transient modulation in brain rhythms in response to task relevant stimuli under physical effort.

An alternative line of investigations have focused on target-locked brain responses during exercise by means of EEG event-related brain potentials (ERPs) as a way to pinpoint the brain correlates of (task relevant) stimulus processing under physical effort [13][14][15][16][17][18]. Here, we take a step further by analysing power spectral changes time-locked to the (task) stimulus instead of ERPs. The event-related spectral perturbation (ERSP) analysis refers to transient decreases or increases in oscillatory brain activity locked to an event, which are thought to reflect the state of synchrony in a population of neurons [19][20][21], and it may provide complementary information to that of the ERPs. The study of ERSP during exercise was achieved by including an oddball task whereby participants had to detect infrequent targets presented among a sequence of frequent distractors (see Yagi et al. [18], for a similar approach although using ERPs).

Our participants exercised at two different intensities, corresponding to the $80 \%$ and $30 \%$ of their $\mathrm{VO}_{2 \max }$ (i.e., maximal aerobic capacity). This selection was motivated by previous evidence pointing to moderate-to-high acute exercise (between the $60 \%$ and $80 \% \mathrm{VO}_{2 \max }$ ) as the key intensity to induce cognitive enhancement [22][23][24]. The $30 \%$ condition was included as the low-intensity exercise baseline (instead of a rest non-exercise condition) to control for potential dual-tasking (i.e., participants were both exercising and performing a cognitive task).

In sum, the current empirical study focused on the tonic oscillatory brain activity and, for the first time, on the event-related brain oscillatory responses during two sessions of acute aerobic exercise at different intensities (moderate-to-high and light) while performing an oddball task. Importantly, taking into account the sparse literature about the brain dynamics during physical exercise we took an exploratory approach (cf. Wagenmakers et al. [25]), with a bottom-up methodology by employing a stepwise cluster-based analysis without prior assumptions on any frequency range or brain localization. 


\section{Material and Methods}

\section{Participants}

We recruited 20 young males with a high level of aerobic fitness (age between 18-31 years old, average age 23.9 years old) from the University of Granada (Spain). All participants met the inclusion criteria of reporting at least 8 hours of cycling or triathlon training per week, normal or corrected to normal vision, reported no neurological, cardiovascular or musculoskeletal disorders and were taking no medication. Note that high-fit cyclists and triathletes were selected because they are capable of maintaining a pedalling cadence at moderate-to-high intensity during long periods of time. Furthermore, they are able to keep a fixed posture over time, which notably reduces EEG movement artifacts. Their fitness level was verified by an incremental effort test (see below). Participants were required to maintain a regular sleep-wake cycle for at least one day before each experimental session and to abstain from stimulating beverages or any intense physical activity 24 hours before each session. All subjects gave written informed consent before the study. The protocol was in accordance with both, the ethical guidelines of the University of Granada, and the Declaration of Helsinki.

\section{Apparatus and materials}

All participants were fitted with a Polar RS800 CX monitor (Polar Electro Öy, Kempele, Finland) to record their heart rate (HR) during the incremental exercise test. We used a ViaSprint $150 \mathrm{P}$ cycle ergometer (Ergoline GmbH, Germany) to induce physical effort and to obtain power values, and a JAEGER Master Screen gas analyser (CareFusion GmbH, Germany) to provide a measure of gas exchange during the effort test. Oddball stimuli were presented on a 21-inch BENQ screen maintaining a fixed distance of $100 \mathrm{~cm}$ between the head of participants and the center of the screen. E-Prime software (Psychology Software Tools, Pittsburgh, PA, USA) was used for stimulus presentation and behavioural data collection.

\section{Fitness Assessments}

Participants came to the laboratory, at least one week before the first experimental session, to provide the informed consent, complete an anthropometric evaluation (height, weight and body mass index $[\mathrm{BMI}]$ ) and to familiarize with the oddball task. Subsequently, they performed an incremental cycle-ergometer test to obtain their maximal oxygen consumption $\left(\mathrm{VO}_{2 \max }\right)$ which was used in the following experimental sessions to adjust the exercise intensity individually. The incremental effort test started with a 3 minutes warm-up at $30 \mathrm{Watts}(\mathrm{W})$, with the power output increasing $10 \mathrm{~W}$ every minute. Each participant set his preferred cadence (between 60-90 $\mathrm{rpm} \cdot \mathrm{min}^{-1}$ ) during the warm-up period and was asked to maintain this cadence during the entire protocol. The test began at $60 \mathrm{~W}$ and was followed by an incremental protocol of $30 \mathrm{~W}$ every 3 minutes. Each step of the incremental protocol consisted of 2 minutes of stabilized load and 1 minute of progressive load increase ( $5 \mathrm{~W}$ every 10 seconds). The oxygen uptake $\left(\mathrm{VO}_{2} \mathrm{ml} \cdot \mathrm{min}^{-1} \cdot \mathrm{kg}^{-1}\right)$, respiratory exchange ratio (RER; i.e., $\mathrm{CO}_{2}$ production $\bullet \mathrm{O}_{2}$ consumption $\left.{ }^{-1}\right)$, relative power output $\left(\mathrm{W} \cdot \mathrm{Kg}^{-1}\right)$ and heart rate $(\mathrm{bpm})$ were continuously recorded throughout the test. 


\section{Experimental sessions}

Participants completed two counterbalanced experimental sessions of approximately $100 \mathrm{~min}$ each. To avoid possible fatigue and/or training effects, visits to the laboratory were scheduled on different days allowing a time interval of 48-72 hours between sessions. On each experimental session, after 10' warm-up on a cycle-ergometer at a power load of $30 \%$ of their individual $\mathrm{VO}_{2 \max }$, participants performed an oddball task for 20 ' while pedalling either at $30 \%$ (light intensity exercise session) or $80 \%$ (moderateintensity exercise session) of their $\mathrm{VO}_{2 \max }$. Upon completion of the oddball task, a 10' cool down period at 30\% of intensity followed (see Table 1). Each participant set his preferred cadence (between 60-90 rpm . $\min ^{-1}$ ) before the warm-up and was asked to maintain this cadence throughout the session in order to match conditions in terms of dual-task demands.

\section{Oddball task}

The visual oddball task was based on that reported in Sawaki and Katayama [26]. The task consisted of a random presentation of three visual stimuli, consisting of a frequent small blue circle (approximately $1.15^{\circ} \times 1.15^{\circ}$ ), a rare big blue circle (approximately $1.30^{\circ} \times 1.30^{\circ}$ ), and a rare red square (approximately $2.00^{\circ} \times 2.00^{\circ}$ ). Small blue circles were considered as standard stimuli (non-target), while big blue circles and red squares were considered as target stimuli. Stimuli were displayed sequentially on the center of the screen on a black background. Each trial started with the presentation of a blank screen in a black background for $1200 \mathrm{~ms}$. Then, the stimulus was presented at a random time interval (between 0 and $800 \mathrm{~ms}$ ) during $150 \mathrm{~ms}$. Participants were instructed to respond to both targets by pressing a button connected to the cycle-ergometer handlebar with the thumb of their dominant hand and to not respond when standard stimuli were shown. Participants were encouraged to respond as accurately as possible. The target stimuli were randomly presented in $20 \%$ of trials (10\% of target $1,10 \%$ target 2 ) and the nontarget stimulus in the remaining $80 \%$ of trials. A total of 600 stimuli were presented. The task lasted for 20 minutes approximately. No breaks were allowed.

\section{EEG recording and analysis}

EEG data were recorded at $1000 \mathrm{~Hz}$ using a 30-channel actiCHamp System (Brain Products $\mathrm{GmbH}$, Munich, Germany) with active electrodes positioned according to the 10-20 EEG International System and referenced to the $\mathrm{Cz}$ electrode. The cap was adapted to individual head size, and each electrode was filled with Signa Electro-Gel (Parker Laboratories, Fairfield, NJ) to optimize signal transduction. Participants were instructed to avoid body movements as much as possible, and to keep their gaze on the center of the screen during the task. Electrode impedances were kept below $10 \mathrm{k} \Omega$. EEG preprocessing was conducted using custom Matlab scripts and the EEGLAB [27] and Fieldtrip [28] Matlab toolboxes. EEG data were resampled at $500 \mathrm{~Hz}$, bandpass filtered offline from 1 and $40 \mathrm{~Hz}$ to remove signal drifts and line noise, and re-referenced to a common average reference. Horizontal electrooculograms (EOG) were recorded by bipolar external electrodes for the offline detection of ocular artifacts. Independent component analysis was used to detect and remove EEG components reflecting eye blinks [29]. 
Spectral power analysis. Electrodes presenting abnormal power spectrum were identified via visual inspection and replaced by spherical interpolation. Processed EEG data from each experimental period (Warm-up, Exercise, Cool Down) were subsequently segmented to 1-s epochs. The spectral decomposition of each epoch was computed using Fast Fourier Transformation (FFT) applying a symmetric Hamming window and the obtained power values were averaged across experimental periods.

ERSP analysis. Task-evoked spectral EEG activity was assessed by computing ERSP in epochs extending from $-500 \mathrm{~ms}$ to $500 \mathrm{~ms}$ time-locked to stimulus onset for frequencies between 4 and $40 \mathrm{~Hz}$. Spectral decomposition was performed using sinusoidal wavelets with 3 cycles at the lowest frequency and increasing by a factor of 0.8 with increasing frequency. Power values were normalized with respect to a $-50 \mathrm{~ms}$ to $0 \mathrm{~ms}$ pre-stimulus baseline and transformed into the decibel scale.

\section{Statistical analysis}

Spectral power main effects of Session (light intensity, Moderate-to-high intensity) were separately tested for significance at each period (Warm-up, Exercise, Cool Down). In the absence of strong a priori hypotheses, we used a stepwise, cluster-based, non-parametric permutation test [30] (Fieldtrip toolbox) without prior assumptions on any frequency range or area of interest. The algorithm performed a t-test for dependent samples on all individual electrodes $\mathrm{x}$ frequencies pairs and clustered samples with positive and negative t-values that exceeded a threshold ( $p<0.05$, two-tailed) based on spatial and spectral adjacency. These comparisons were performed for each frequency bin of $1 \mathrm{~Hz}$ and for each electrode. Cluster-level statistics were then calculated by taking the sum of the t-values within each cluster. The trials from the two datasets (light intensity, Moderate-to-high intensity) were randomly shuffled and the maximum cluster-level statistic for these new shuffled datasets was calculated. The above procedure was repeated 5000 times to estimate the distribution of maximal cluster-level statistics obtained by chance. The proportion of random partitions that resulted in a larger test statistic than the original one determined the two-tailed Monte-Carlo p-value.

In addition, ERSP main effects of Condition (light intensity, Moderate-to-high intensity) for each stimulus (target 1, target 2 and standard) were also analysed by applying the cluster-based permutation test. In order to reduce the possibility that the type II error rate was inflated by multiple comparisons correction, we grouped data into four frequency bands: Theta (4-8 Hz), Alpha (8-14 Hz), lower Beta $(14-20 \mathrm{~Hz})$ and upper Beta $1(20-40 \mathrm{~Hz})$. Note that the time window of interest in target trials was restricted to the first $300 \mathrm{~ms}$ after target onset in order to avoid an overlap with behavioural responses based on average reaction time (RT). The time window of interest for standard trials was fixed to the first $500 \mathrm{~ms}$ after the stimulus onset.

Behavioural data from both experiments were analysed using a within-participants factor of Session (light intensity, Moderate-to-high intensity) for RT and accuracy (ACC) as dependent variables. All analyses were completed using statistical non-parametric permutation tests with a Monte Carlo approach [31][32]. 


\section{Results}

\section{Spectral power analysis}

The analysis of tonic spectral power showed a significant main effect of Session for the exercise period (all $p s<.02$ ). Two positive clusters (frequency-localization) were found and were statistically significant: one global cluster (25 electrodes) in low frequencies (1-5 Hz), cluster $p<.001$, and one parieto-occipital cluster $(11$ electrodes) in fast frequencies $(8-40 \mathrm{~Hz})$, cluster $p=.017$. The analysis revealed an overall increase in the power of frequencies during the moderate-to-high intensity exercise period in comparison to light intensity (see Fig. 1). There were no statistically significant between-session differences in warm-up and cool down periods (all cluster $p s \geq .1$ ).

A)

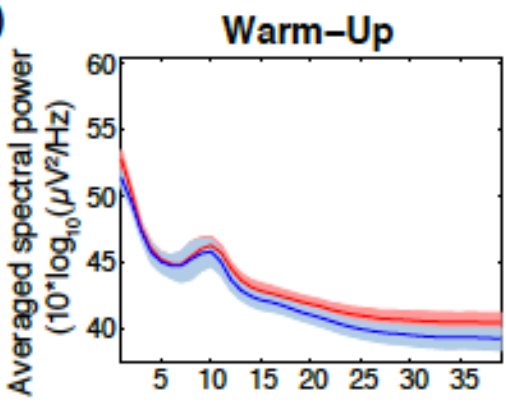

Exercise

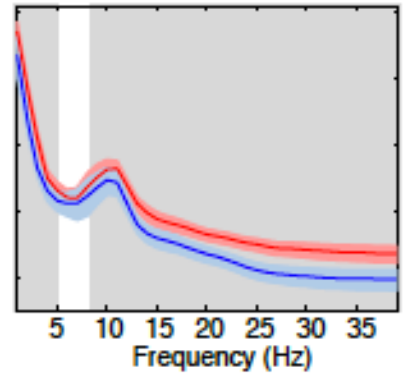

Cool Down

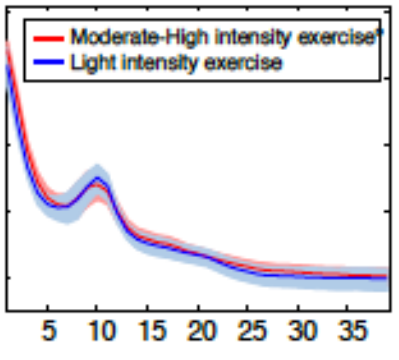

B)

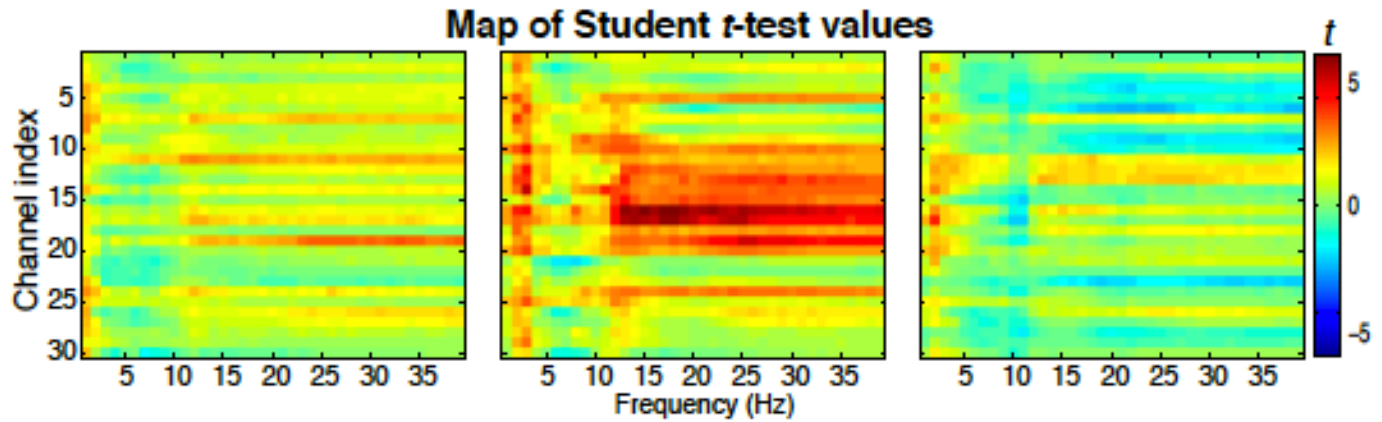

C)

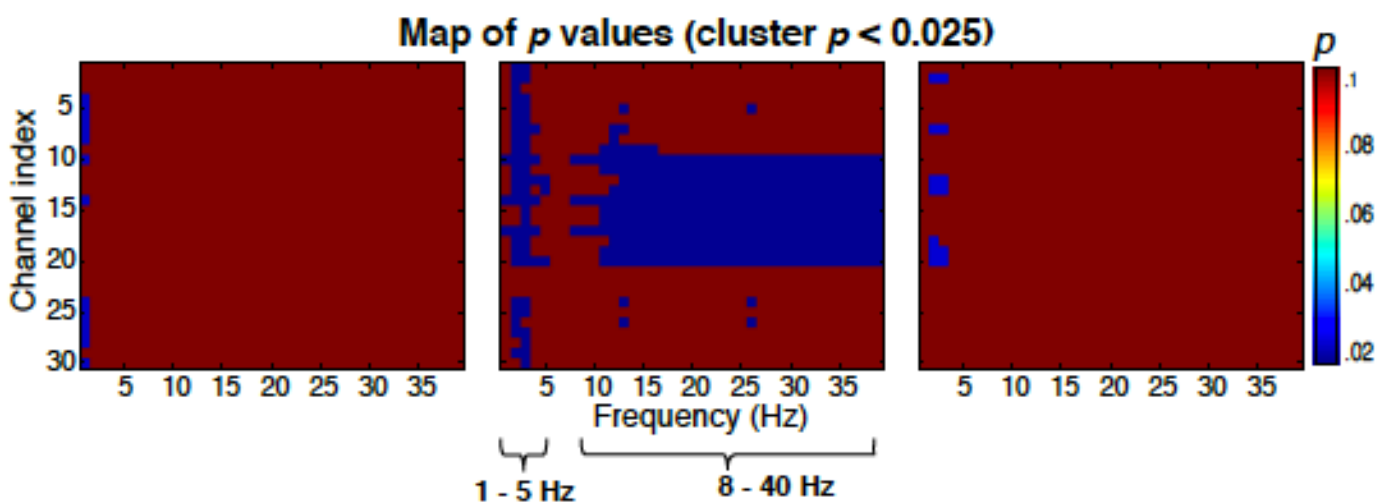

D)

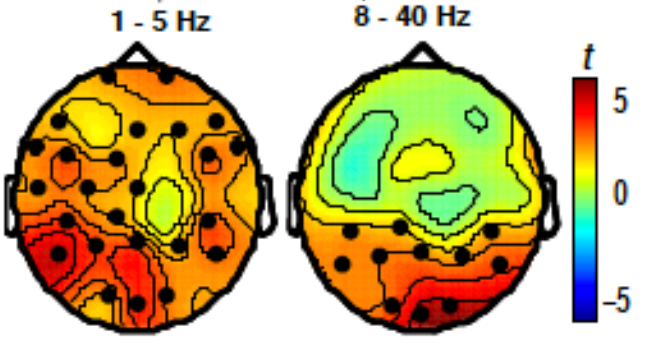

Figure 1. Modulation of brain power spectrum as a function of exercise intensity. (A) Differences in the averaged EEG power spectrum across subjects between moderate-to-high intensity (red lines) and 
light intensity (blue lines) exercise at the three experimental periods (warm-up, exercise and cool down). Red and blue shaded areas represent $95 \%$ confidence intervals. Statistically significant differences are marked by grey area (B) Parametric paired t-test maps comparing the relative power across frequency bands (x-axes) and channels (y-axes) during moderate-to-high intensity and light intensity exercise at warm-up, exercise and cool down (blue: decreases; red: increases). (C) Each image illustrates the statistical significance ( $p$ values) of the $t$-maps depicting only the significant clusters with $p<0.025$. (D) Topographies depict t-test distribution in all electrodes, showing the spatial characteristics of the increase in power of low frequencies across the whole surface localization during moderate-to-high exercise, and the increase in high frequencies in parieto-occipital areas during moderate-to-high exercise. No significant between-intensity differences were found at warm-up and cool down.

\section{ERSP analysis}

Fig. 2 shows the time-locked oscillatory activity of the oddball task during both exercise periods. The analysis of the ERSP revealed a significant main effect of session for standard trials in alpha and lower beta bands (all ps <.015). The alpha band analysis revealed only an occipital positive cluster (4 electrodes) between 150-500 ms after the onset of the standard, $p=.014$, with higher alpha spectral power during moderate-to-high intensity exercise compared to light intensity (see Fig 3a). The lower Beta band analysis showed only a parieto-occipital significant positive cluster ( 8 electrodes) between 300-500 $\mathrm{ms}$ after the onset of the standard, $p=.009$. Lower Beta frequency band exhibited a higher spectral power in the moderate-to-high intensity session than in the light intensity session (see Fig 3b).

A)

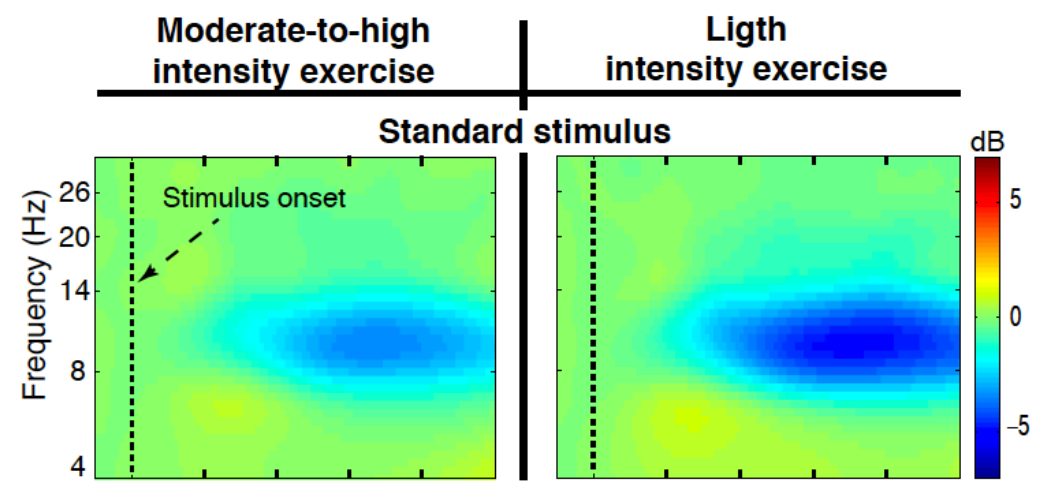

B)

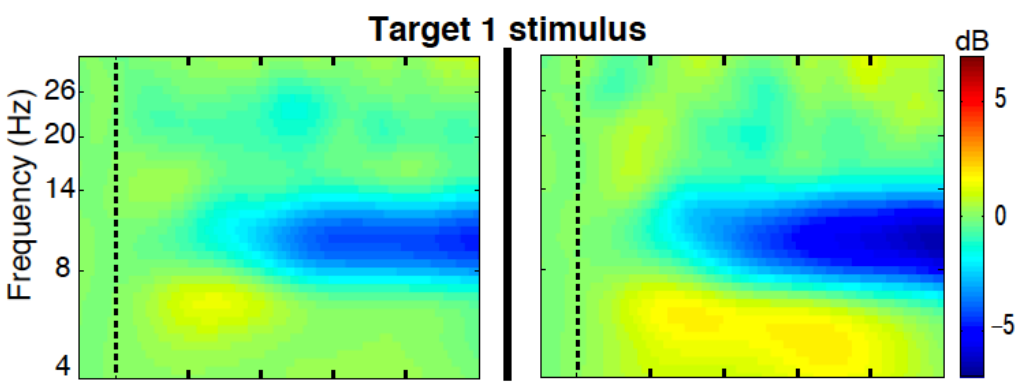

C)

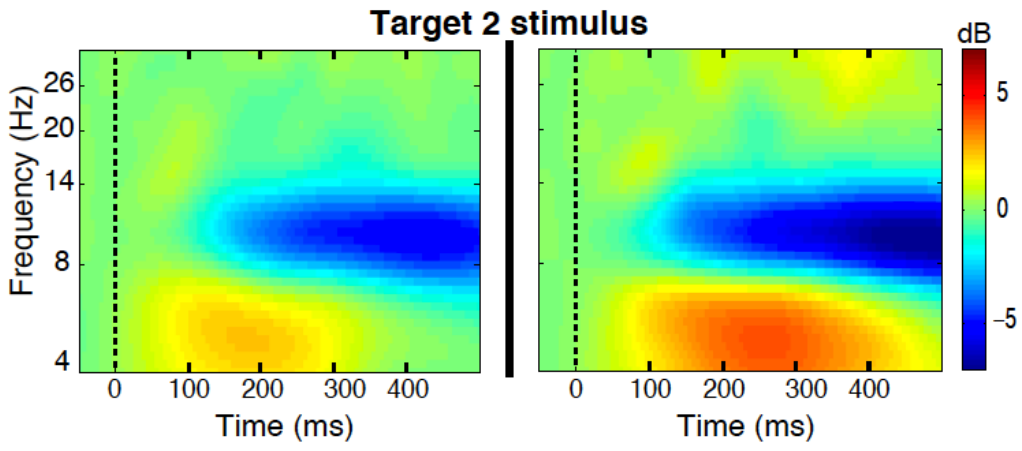


bioRxiv preprint doi: https://doi.org/10.1101/201749; this version posted October 11,2017 . The copyright holder for this preprint (which was not certified by peer review) is the author/funder, who has granted bioRxiv a license to display the preprint in perpetuity. It is made available under aCC-BY-NC-ND 4.0 International license.

Figure 2. Event-related spectral perturbation of oddball task during exercise. Time-locked spectral power averaged over the occipital channels (Oz, O1 and O2) during moderate-to-high intensity (left column) and light intensity (right column) exercise for all stimuli (standard, target 1 and target 2). Each panel illustrates time-frequency power across time (x-axes) and frequency (y-axes) during moderateintensity and light-intensity exercise (blue: decreases; red: increases).

The target 2 trials analysis revealed a significant main effect of Session in the theta band $(p<$ $.01)$. Two negative clusters (time-localization) were found. Only the largest global negative cluster (7 electrodes) between 190-250 ms after the onset of the target was significant, cluster $p=.004$. Target 2 trials (red squares) during moderate-to-high intensity exercise session evoked lesser theta power than during light intensity exercise condition (see Fig 3c). The analysis of the other frequency bands for target 1 , target 2 and standard trials did not yield significant effects (all $p s \geq$. 05).

A)

Alpha power modulation during exercise in Standard trials
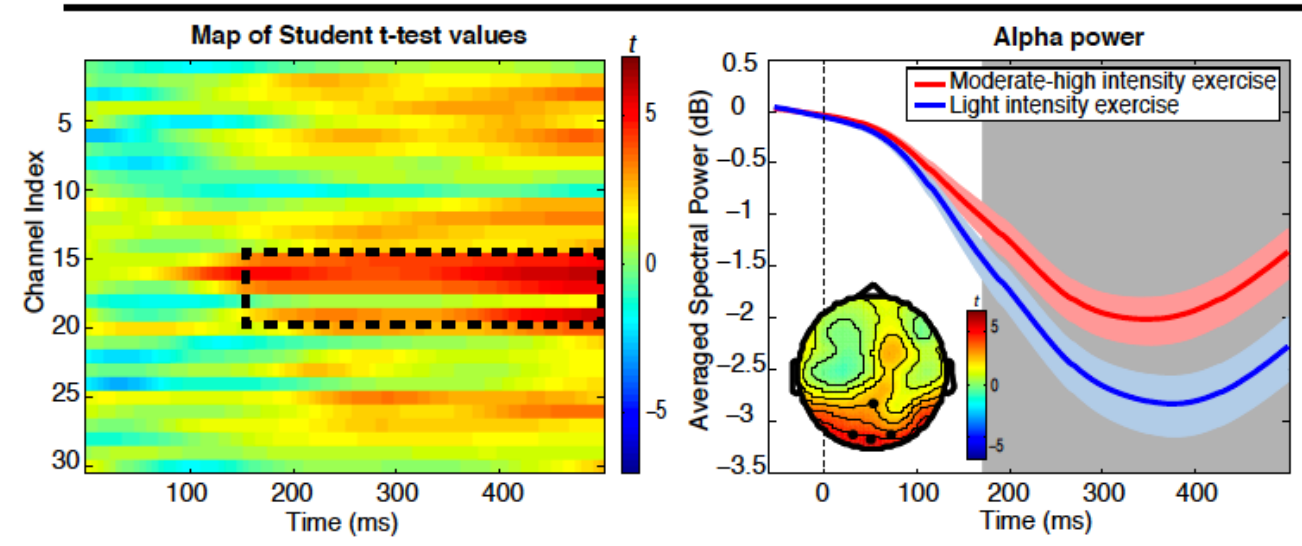

B)

Lower Beta power modulation during exercise in Standard trials
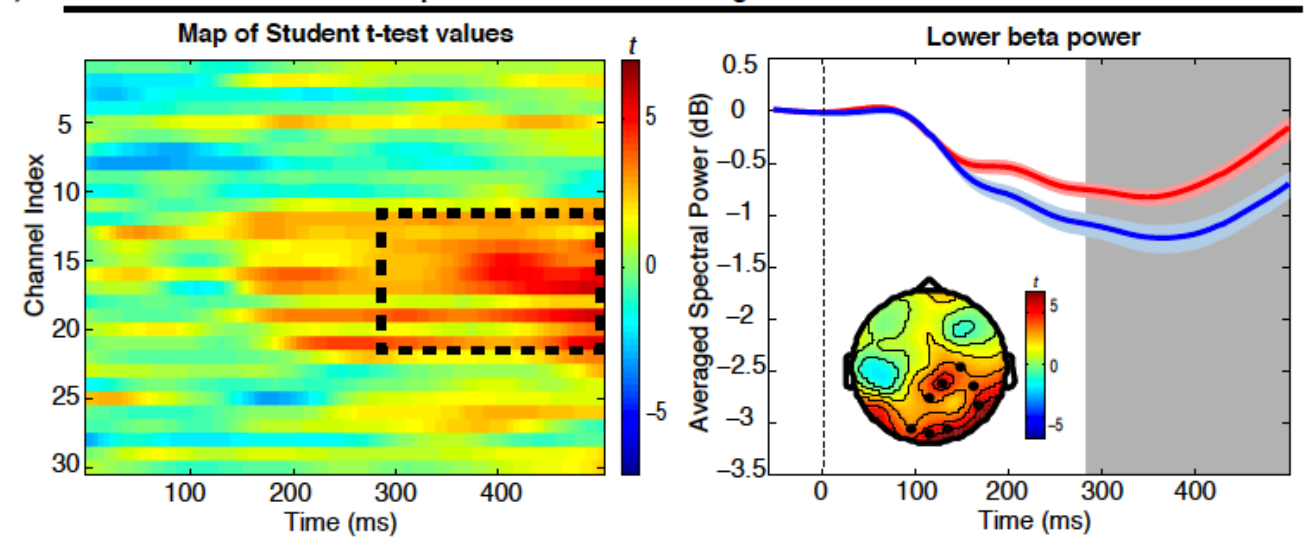

C)

Theta power modulation during exercise in Target 2 trials
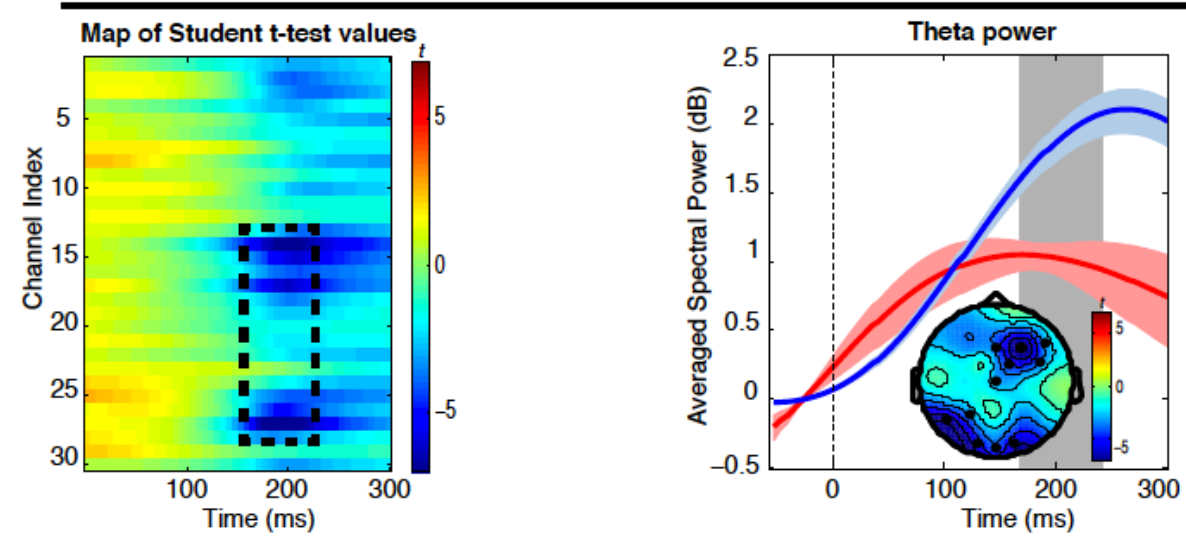
Figure 3. Event-related spectral perturbation significant main effects of session. (A) Alpha frequency band [8-14 Hz] parametric paired t-test maps comparing the averaged spectral power across subjects over time (x-axes) and frequency (y-axes) during moderate-to-high intensity and light intensity exercise in standard trials. The enclosed areas denote significant clusters of channels and time with $\mathrm{p}<$ 0.025. Right panel shows alpha power across time at the occipital-parietal cluster (4 electrodes) in standard trials. The shaded area represents the latency range where significant differences between exercise sessions were found. Red and blue shaded areas represent 95\% confidence intervals. The topography depicts t-test distribution across surface localization, showing the spatial characteristics of the higher power suppression of alpha in parieto-occipital electrodes during moderate-to-high exercise. (B) Parametric paired t-test maps comparison between moderate-to-high exercise and light exercise in lower beta frequency band [14-20 Hz] to standard trials. Right panel shows lower beta power across time at the parieto-occipital cluster (8 electrodes) in standard trials. (C) Theta frequency band [4-8 Hz] parametric paired t-test maps comparing moderate-to-high intensity and light intensity exercise in target 2 trials (red squares). Right panel shows theta power across time at the globally-localized cluster ( 7 electrodes) in target 2 trials. Note that the time window of interest in target trials was restricted to the first $300 \mathrm{~ms}$ in order to avoid neural activity overlapping with behavioural responses. The analysis of the other frequency bands for standard, target 1 , and target 2 stimuli did not yield significant effects.

\section{Behavioural Performance}

Table 1 provides mean and 95\% confidence intervals of behavioural measures as a function of session. Analysis of response RTs and ACC did not reveal statistically significant differences between sessions, (all ps $>.05)$.

Table 1. Mean and 95\% confidence intervals of descriptive exercise-intensity parameters and behavioural performance for the moderate-to-high intensity and low intensity sessions.

\begin{tabular}{|c|c|c|c|c|}
\hline & \multicolumn{2}{|c|}{$\begin{array}{l}\text { Moderate-to-high intensity } \\
\qquad\left(80 \% \mathrm{VO}_{2 \max }\right)\end{array}$} & \multicolumn{2}{|c|}{$\begin{array}{l}\text { Light intensity } \\
\left(30 \% \mathrm{VO}_{2 \max }\right)\end{array}$} \\
\hline \multicolumn{5}{|c|}{ Exercise period parameters } \\
\hline Mean power load (W) & \multicolumn{2}{|c|}{$233.4 \pm 242.8$} & \multicolumn{2}{|c|}{$87.5 \pm 90.8$} \\
\hline $\begin{array}{l}\text { Mean relative power } \\
\operatorname{load}(\mathrm{W} / \mathrm{kg})\end{array}$ & \multicolumn{2}{|c|}{$3.0 \pm 3.2$} & \multicolumn{2}{|c|}{$1.1 \pm 1.2$} \\
\hline \multicolumn{5}{|c|}{ Behavioural performance } \\
\hline & $\operatorname{ACC}(\%)$ & RT (ms) & $\operatorname{ACC}(\%)$ & RT (ms) \\
\hline Standard & $.99 \pm 1.0$ & --- & $1.0 \pm 1.0$ & --- \\
\hline Target 1 & $.90 \pm .9$ & $315.7 \pm 352.3$ & $.88 \pm .9$ & $320.6 \pm 363.8$ \\
\hline Target 2 & $.98 \pm 1.0$ & $234.7 \pm 256.5$ & $.97 \pm 1.0$ & $236.9 \pm 260.2$ \\
\hline
\end{tabular}




\section{Discussion}

In the present study, we investigated the oscillatory brain activity of young adults, during a single bout of aerobic exercise (cycling) at $80 \%$ of maximum aerobic capacity compared to a light intensity exercise (control) session while performing a visual oddball task. We found that acute exercise at moderate-to-high intensity induced changes in oscillatory brain activity at the tonic and transient (event-related) level with respect to light intensity.

The overall power increase across the frequency spectrum during moderate-to-high intensity exercise with respect to light intensity is in line with the results of the only meta-analysis to date that has addressed this issue [12]. Indeed, Crabbe and Dishman [12] found no evidence of the selective effect of exercise on alpha frequency band at frontal localizations suggested by previous empirical research $[9][10][11][33]$. Interestingly, the between-intensity differences were unspecific of surface localization in slow frequencies, while in faster frequencies the differences arose from parieto-occipital sites. These results partially contradict previous studies that have shown changes in oscillatory brain activity during exercise localized in anterior sites [9][34].

Several studies have suggested that the theta band is selectively enhanced by the presentation of novel stimuli, linking it to the orienting responses associated with novelty processing [35][36]. Moreover, alpha activity suppression has been associated with cognitive engagement to the task [37][38]during oddball paradigms similar to the one uses in the present study [39][40]. Thus, the present theta and alpha results might represent an attentional resources regulation during stimulus processing in order to maintain optimal task performance. In line with this interpretation, and given that task behavioural performance was not significantly different between sessions, one could argue that the reduced theta power to target 2 trials (the more salient stimuli) and the lower suppression of alpha and lower beta bands during the moderate-to-high intensity exercise session with respect to the light intensity session, was a sign of brain efficiency. Results from previous ERP studies during a single bout of exercise have also pointed to processing efficiency during moderate-to-high intensity exercise [17][41][42]. At this point, it is important to note that both exercise sessions were matched in terms of dual-task demands. Participants were instructed to maintain the pedaling cadence constant between 60 and $90 \mathrm{rpm}$ in both sessions, therefore any variation in brain functioning was due to the physiological changes induced by the particular exercise intensity.

Exercising elicits a wide set of physiological changes such as increases in core temperature, cortical blood flow, heart rate, and catecholamine concentration [43] which have generally been recognized as a potential mechanism underlying the acute exercise effect on brain function. Interestingly, we found a higher global increase of oscillatory brain activity during the moderate-to-high intensity session than the light intensity session. This latter result is consistent with recent accounts that have linked acute exercise to enhanced activation/arousal (that relates to the overall activation/excitability of cortical neurons [44][45]). However, the ERSP results in our study suggest that the effect of acute exercise cannot be explained as a mere overall increase of oscillatory brain activity but to a specific way of brain functioning during exercise (at moderate-to-high intensity).

To conclude, the present study contributes to further understanding of how the brain works during acute exercise and the underlying neural mechanisms of the positive relationship between physical 
exercise, brain function and cognition in young adults. Importantly, a profound knowledge of the factors that support this beneficial relationship is especially critical for public health, as a sedentary lifestyle has been linked to a series of chronic diseases and to a deterioration of cognitive function and performance in many daily life activities.

\section{Acknowledgments}

This work was supported by the "Ministerio de Economía y Competitividad" (grant numbers PSI2013-46385-P and PSI2016-75956-P) and the "Junta de Andalucía" (grant number SEJ-6414). P.Ch.I. acknowledges support from W. M. Keck Foundation, and the Office of Naval Research (ONR Grant No. 000141010078). The funders had no role in study design, data collection and analysis, decision to publish, or preparation of the manuscript. We thank to all the participants who took part in the experiment.

\section{References}

1. McArdle, W.D., Katch, F.I., and Katch, V.L. (2010). Exercise Physiology: Nutrition, Energy, and Human Performance (Lippincott Williams \& Wilkins).

2. Karasik, R., Sapir, N., Ashkenazy, Y., Ivanov, P.C., Dvir, I., Lavie, P., and Havlin, S. (2002). Correlation differences in heartbeat fluctuations during rest and exercise. Physical Review E 66, 062902.

3. Ashkenazy, Y., Hausdorff, J.M., Ivanov, P.C., and Stanley, H.E. (2002). A stochastic model of human gait dynamics. Physica A: Statistical Mechanics and its Applications 316, 662-670.

4. Ivanov, P.C., Hu, K., Hilton, M.F., Shea, S.A., and Stanley, H.E. (2007). Endogenous circadian rhythm in human motor activity uncoupled from circadian influences on cardiac dynamics. Proceedings of the National Academy of Sciences 104, 20702-20707.

5. Ivanov, P.C., Ma, Q.D., Bartsch, R.P., Hausdorff, J.M., Amaral, L.A.N., Schulte-Frohlinde, V., Stanley, H.E., and Yoneyama, M. (2009). Levels of complexity in scale-invariant neural signals. Physical Review E 79, 041920.

6. Baillet, M., Dilharreguy, B., Pérès, K., Dartigues, J.-F., Mayo, W., and Catheline, G. (2017). Activity/rest cycle and disturbances of structural backbone of cerebral networks in aging. NeuroImage $146,814-820$.

7. Walsh, V. (2014). Is sport the brain's biggest challenge? Current biology 24, R859-R860.

8. Johansen-Berg, H., and Duzel, E. (2016). Neuroplasticity: Effects of Physical and Cognitive activity on brain structure and function. NeuroImage 131, 1-3.

9. Kubitz, K.A., and Pothakos, K. (1997). Does aerobic exercise decrease brain activation? Journal of Sport and Exercise Psychology 19, 291-301.

10. Boutcher, S. (1993). Emotion and aerobic exercise. Handbook of research on sport psychology, 799-814.

11. Petruzzello, S.J., Landers, D.M., Hatfield, B.D., Kubitz, K.A., and Salazar, W. (1991). A MetaAnalysis on the Anxiety-Reducing Effects of Acute and Chronic Exercise: Outcomes and Mechanisms. Sports Medicine 11, 143-182.

12. Crabbe, J.B., and Dishman, R.K. (2004). Brain electrocortical activity during and after exercise: A quantitative synthesis. Psychophysiology 41, 563-574.

13. Bullock, T., Elliott, J.C., Serences, J.T., and Giesbrecht, B. (2017). Acute Exercise Modulates Feature-selective Responses in Human Cortex. Journal of Cognitive Neuroscience 29, 605-618.

14. Olson, R.L., Chang, Y.-K., Brush, C.J., Kwok, A.N., Gordon, V.X., and Alderman, B.L. (2016). Neurophysiological and behavioral correlates of cognitive control during low and moderate intensity exercise. NeuroImage 131, 171-180.

15. Bullock, T., Cecotti, H., and Giesbrecht, B. (2015). Multiple stages of information processing are modulated during acute bouts of exercise. Neuroscience 307, 138-150.

16. Pontifex, M.B., Parks, A.C., Henning, D.A., and Kamijo, K. (2015). Single bouts of exercise selectively sustain attentional processes. Psychophysiology 52, 618-625.

17. Grego, F., Vallier, J.-M., Collardeau, M., Bermon, S., Ferrari, P., Candito, M., Bayer, P., Magnié, M.-N., and Brisswalter, J. (2004). Effects of long duration exercise on cognitive function, blood glucose, and counterregulatory hormones in male cyclists. Neuroscience Letters 364, 76-80.

18. Yagi, Y., Coburn, K.L., Estes, K.M., and Arruda, J.E. (1999). Effects of aerobic exercise and gender on visual and auditory P300, reaction time, and accuracy. European journal of applied physiology 
and occupational physiology 80, 402-408.

19. Klimesch, W., Sauseng, P., Hanslmayr, S., Gruber, W., and Freunberger, R. (2007). Eventrelated phase reorganization may explain evoked neural dynamics. Neuroscience \& Biobehavioral Reviews 31, 1003-1016.

20. Pfurtscheller, G. (1977). Graphical display and statistical evaluation of event-related desynchronization (ERD). Electroencephalography and clinical neurophysiology 43, 757-760.

21. Pfurtscheller, G. (1992). Event-related synchronization (ERS): an electrophysiological correlate of cortical areas at rest. Electroencephalography and clinical neurophysiology 83, 62-69.

22. González-Fernandez, F., Etnier, J.L., Zabala, M., and Sanabria, D. (2017). Vigilance performance during acute exercise. International Journal of Sport Psychology.

23. Chang, Y.-K., and Etnier, J.L. (2009). Exploring the dose-response relationship between resistance exercise intensity and cognitive function. Journal of sport \& exercise psychology 31, 640.

24. Brisswalter, J., Collardeau, M., and René, A. (2002). Effects of acute physical exercise characteristics on cognitive performance. Sports Medicine 32, 555-566.

25. Wagenmakers, E.J., Wetzels, R., Borsboom, D., van der Maas, H.L.J., and Kievit, R.A. (2012). An Agenda for Purely Confirmatory Research. Perspectives on Psychological Science 7, 632-638.

26. Sawaki, R., and Katayama, J.I. (2007). Difficulty of discrimination modulates attentional capture for deviant information. Psychophysiology 44, 374-382.

27. Delorme, A., and Makeig, S. (2004). EEGLAB: an open source toolbox for analysis of singletrial EEG dynamics including independent component analysis. Journal of Neuroscience Methods 134, 921.

28. Oostenveld, R., Fries, P., Maris, E., and Schoffelen, J.-M. (2011). FieldTrip: Open Source Software for Advanced Analysis of MEG, EEG, and Invasive Electrophysiological Data. Computational Intelligence and Neuroscience 2011, 1-9.

29. Hoffmann, S., and Falkenstein, M. (2008). The Correction of Eye Blink Artefacts in the EEG: A Comparison of Two Prominent Methods. PLoS ONE 3, e3004.

30. Maris, E., and Oostenveld, R. (2007). Nonparametric statistical testing of EEG- and MEG-data. Journal of Neuroscience Methods 164, 177-190.

31. Ernst, M.D. (2004). Permutation Methods: A Basis for Exact Inference. Statist. Sci. 19, 676-

685.

32. Pesarin, F., and Salmaso, L. (2010). The permutation testing approach: a review. Statistica 70, 481.

33. Petruzzello, S.J., Hall, E.E., and Ekkekakis, P. (2001). Regional brain activation as a biological marker of affective responsivity to acute exercise: influence of fitness. Psychophysiology 38, 99-106.

34. Bailey, S.P., Hall, E.E., Folger, S.E., and Miller, P.C. (2008). Changes in EEG during graded exercise on a recumbent cycle ergometer. Journal of sports science \& medicine 7, 505.

35. Demiralp, T., Ademoglu, A., Comerchero, M., and Polich, J. (2001). Wavelet analysis of P3a and P3b. Brain topography 13, 251-267.

36. Demiralp, T., Ademoglu, A., Istefanopulos, Y., Başar-Eroglu, C., and Başar, E. (2001). Wavelet analysis of oddball P300. International journal of psychophysiology 39, 221-227.

37. Klimesch, W., Sauseng, P., and Hanslmayr, S. (2007). EEG alpha oscillations: The inhibition?timing hypothesis. Brain Research Reviews 53, 63-88.

38. Jensen, O., and Mazaheri, A. (2010). Shaping Functional Architecture by Oscillatory Alpha Activity: Gating by Inhibition. Frontiers in Human Neuroscience 4. Available at: http://journal.frontiersin.org/article/10.3389/fnhum.2010.00186/abstract [Accessed August 10, 2017].

39. Yordanova, J., Kolev, V., and Polich, J. (2001). P300 and alpha event-related desynchronization (ERD). Psychophysiology 38, 143-152.

40. Yordanova, J., and Kolev, V. (1998). Event-related alpha oscillations are functionally associated with P300 during information processing. Neuroreport 9, 3159-3164.

41. Pontifex, M.B., and Hillman, C.H. (2007). Neuroelectric and behavioral indices of interference control during acute cycling. Clinical Neurophysiology 118, 570-580.

42. Yagi, Y., Coburn, K.L., Estes, K.M., and Arruda, J.E. (1999). Effects of aerobic exercise and gender on visual and auditory P300, reaction time, and accuracy. European journal of applied physiology and occupational physiology 80, 402-408.

43. McMorris, T., and Hale, B.J. (2015). Is there an acute exercise-induced physiological/biochemical threshold which triggers increased speed of cognitive functioning? A metaanalytic investigation. Journal of Sport and Health Science 4, 4-13.

44. Langner, R., and Eickhoff, S.B. (2013). Sustaining attention to simple tasks: A meta-analytic review of the neural mechanisms of vigilant attention. Psychological Bulletin 139, 870-900.

45. Oken, B.S., Salinsky, M.C., and Elsas, S.M. (2006). Vigilance, alertness, or sustained attention: physiological basis and measurement. Clinical Neurophysiology 117, 1885-1901. 
A)
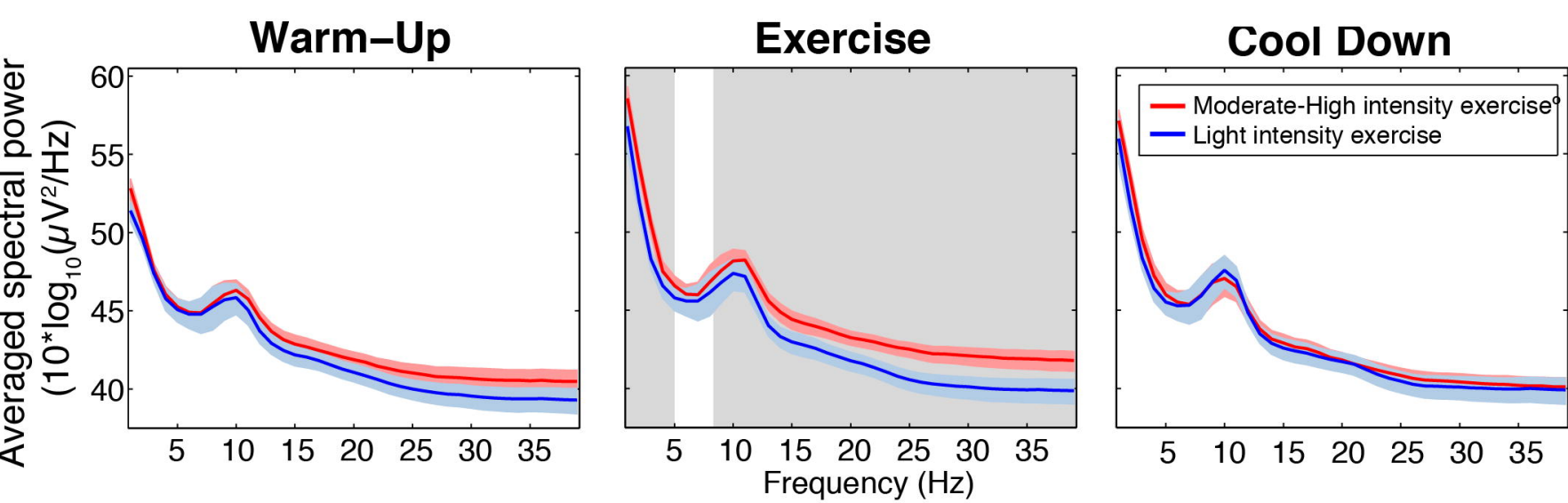

B)

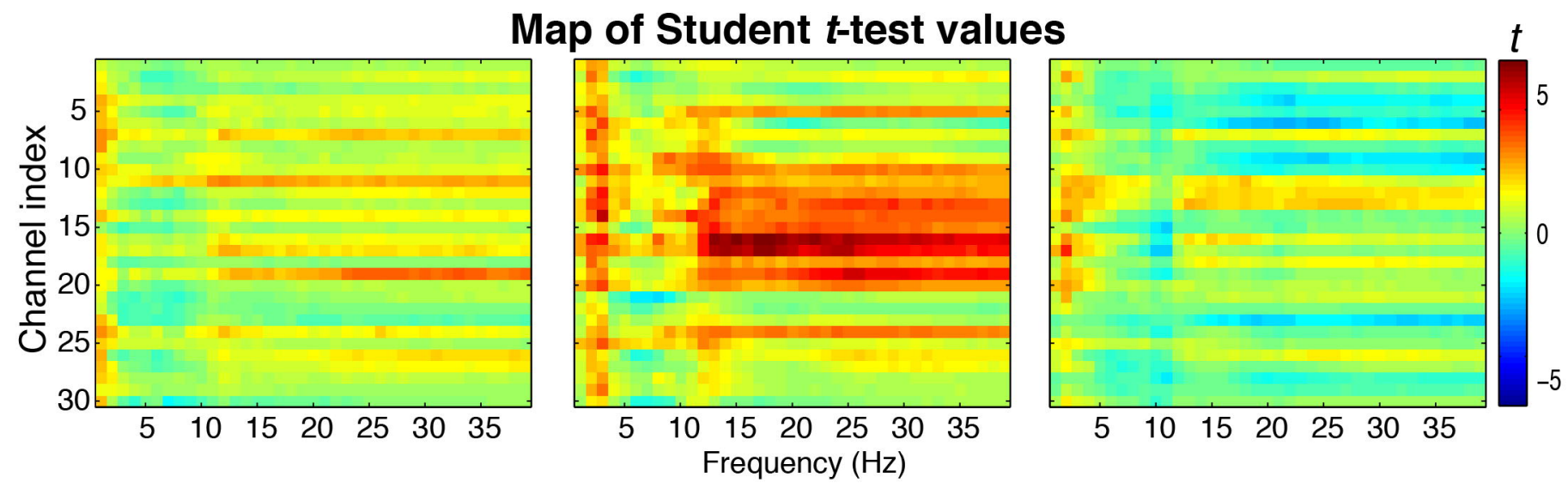

C)

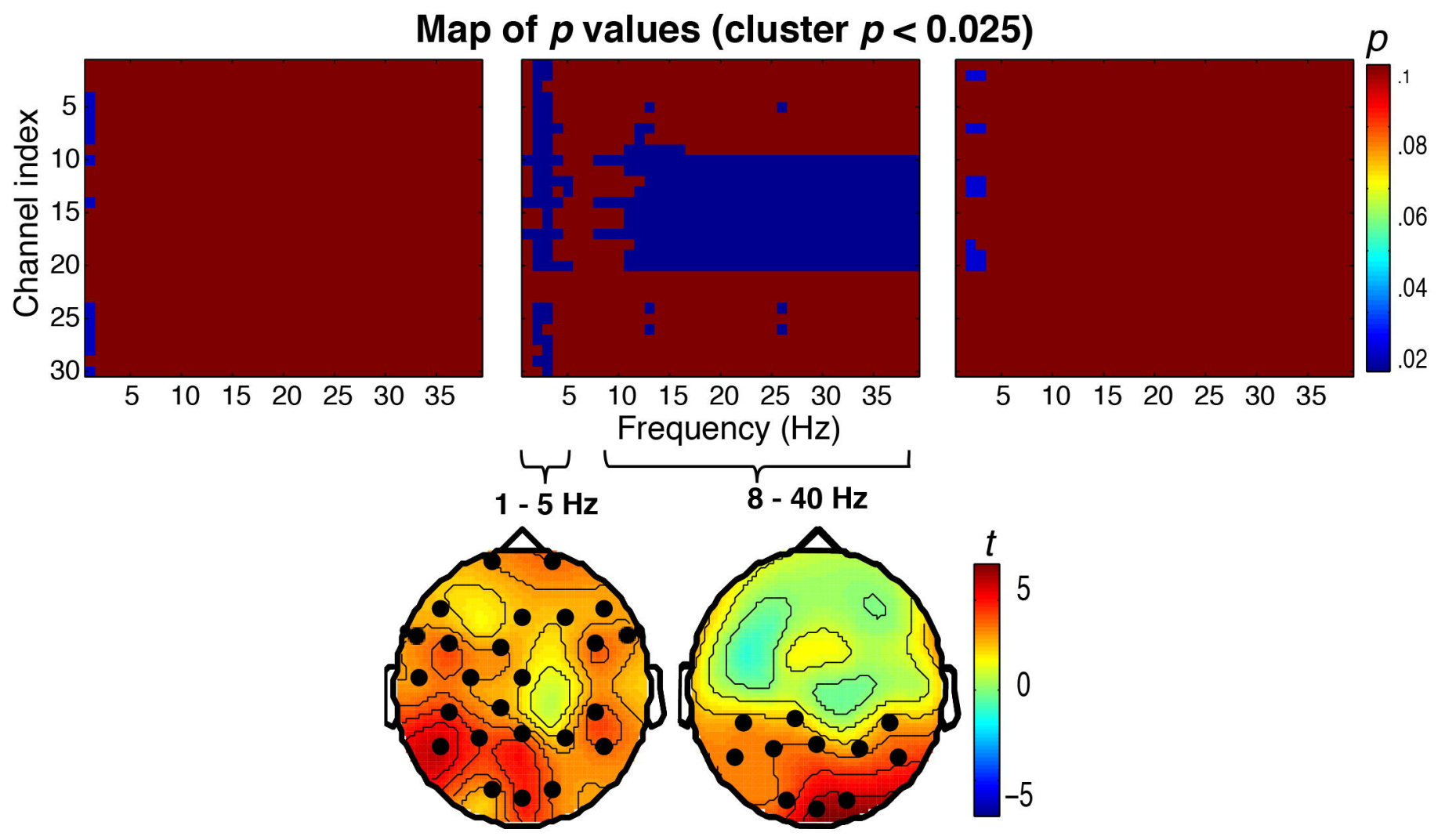


Moderate-to-high

Ligth

A)

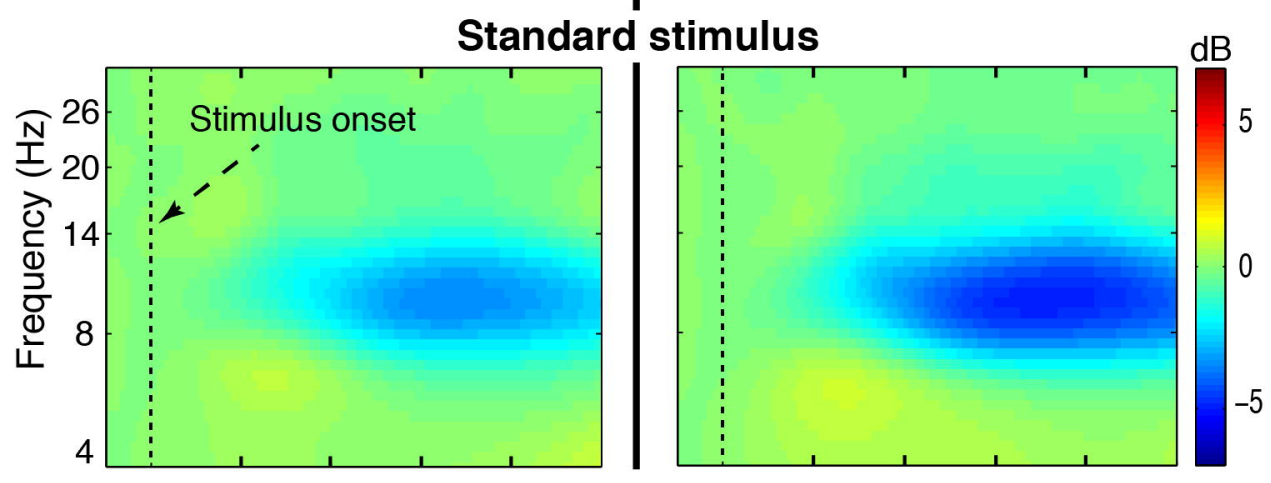

B)

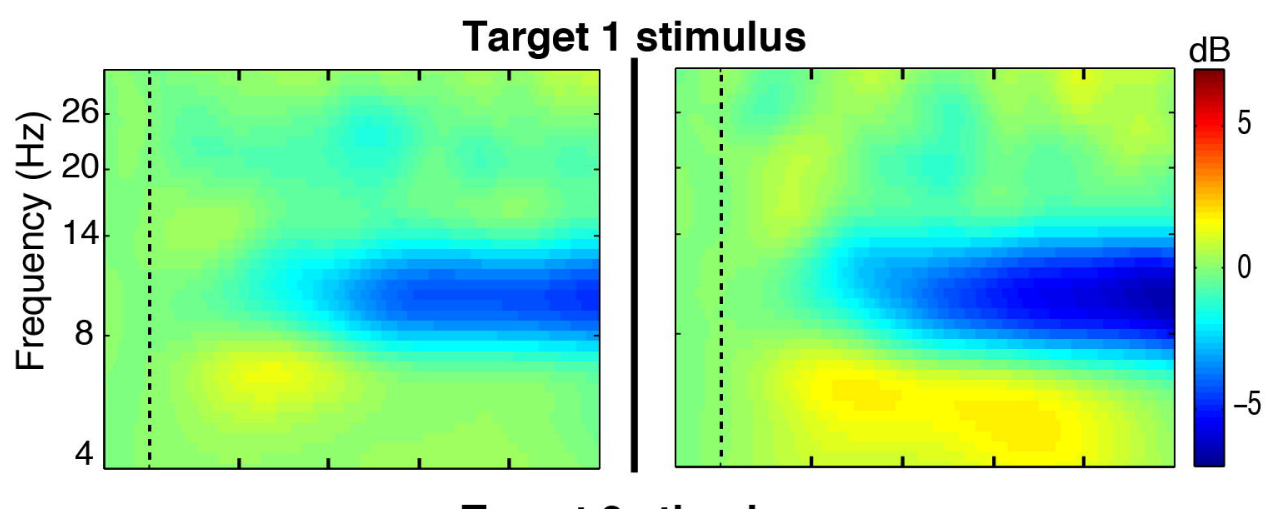

C)

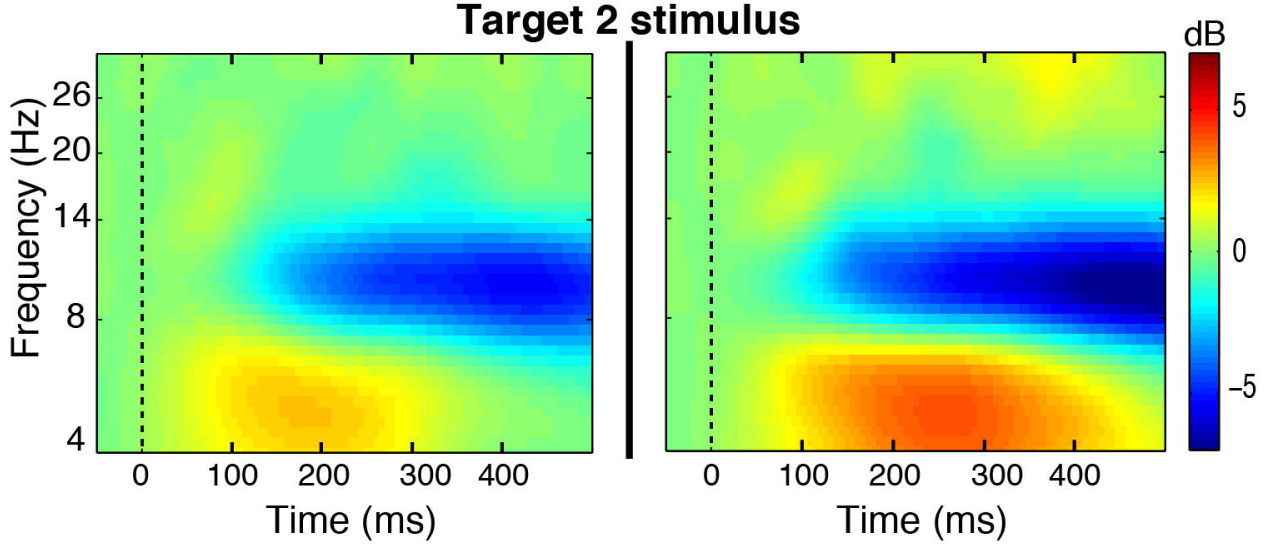


Alpha power modulation during exercise in Standard trials
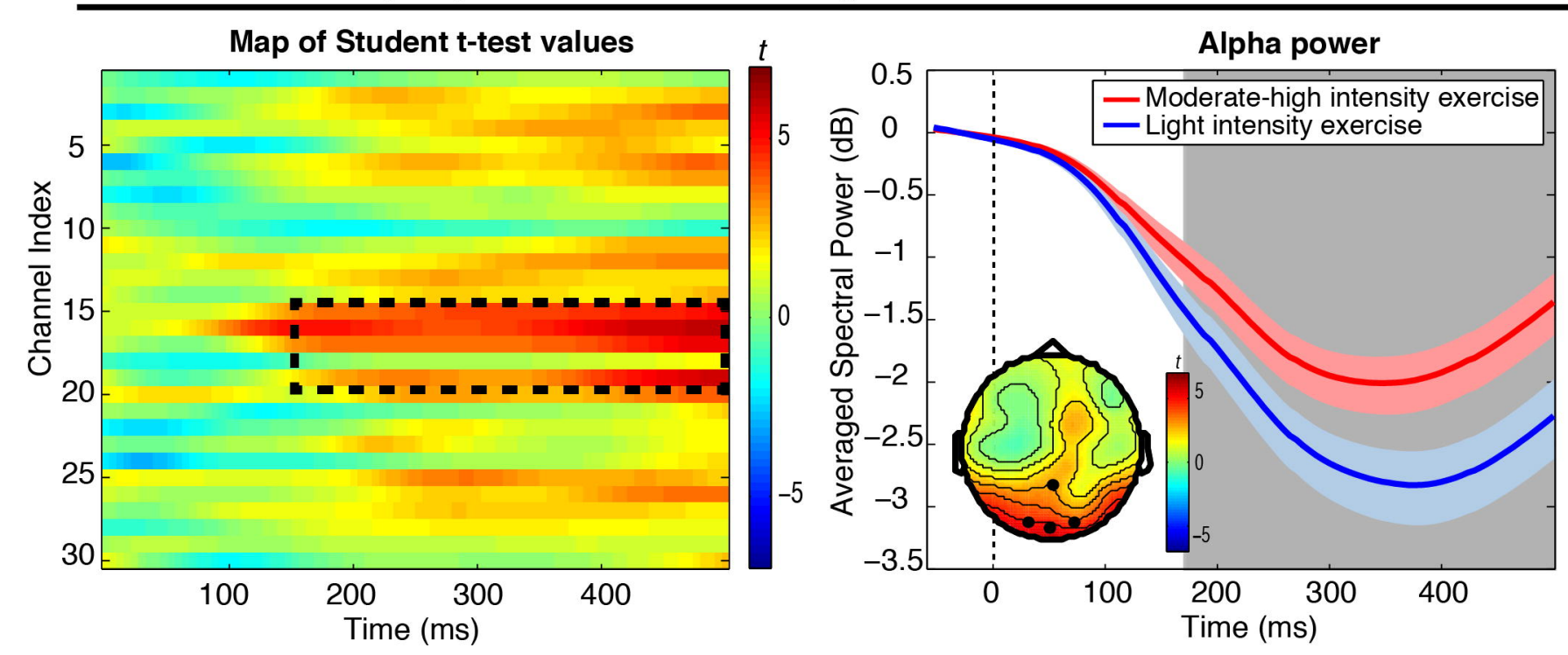

B) Lower Beta power modulation during exercise in Standard trials
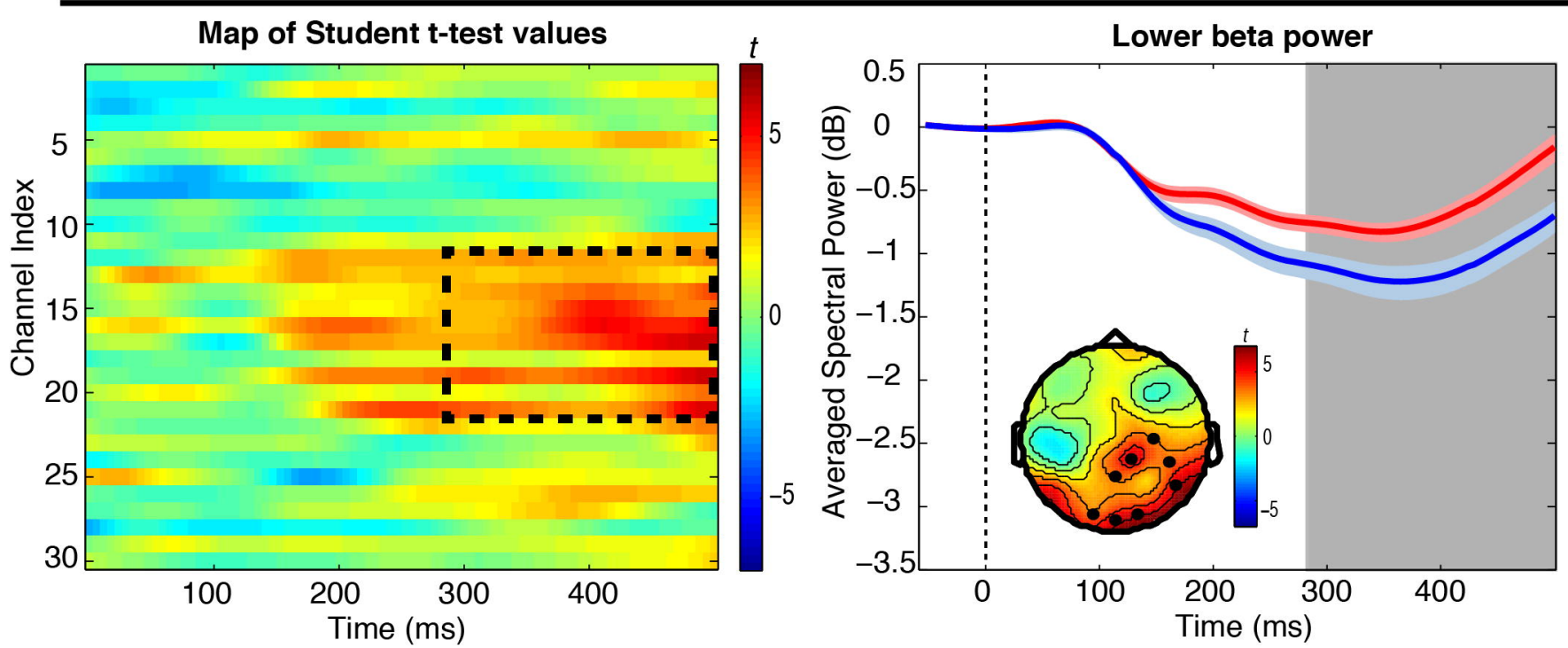

C) Theta power modulation during exercise in Target 2 trials
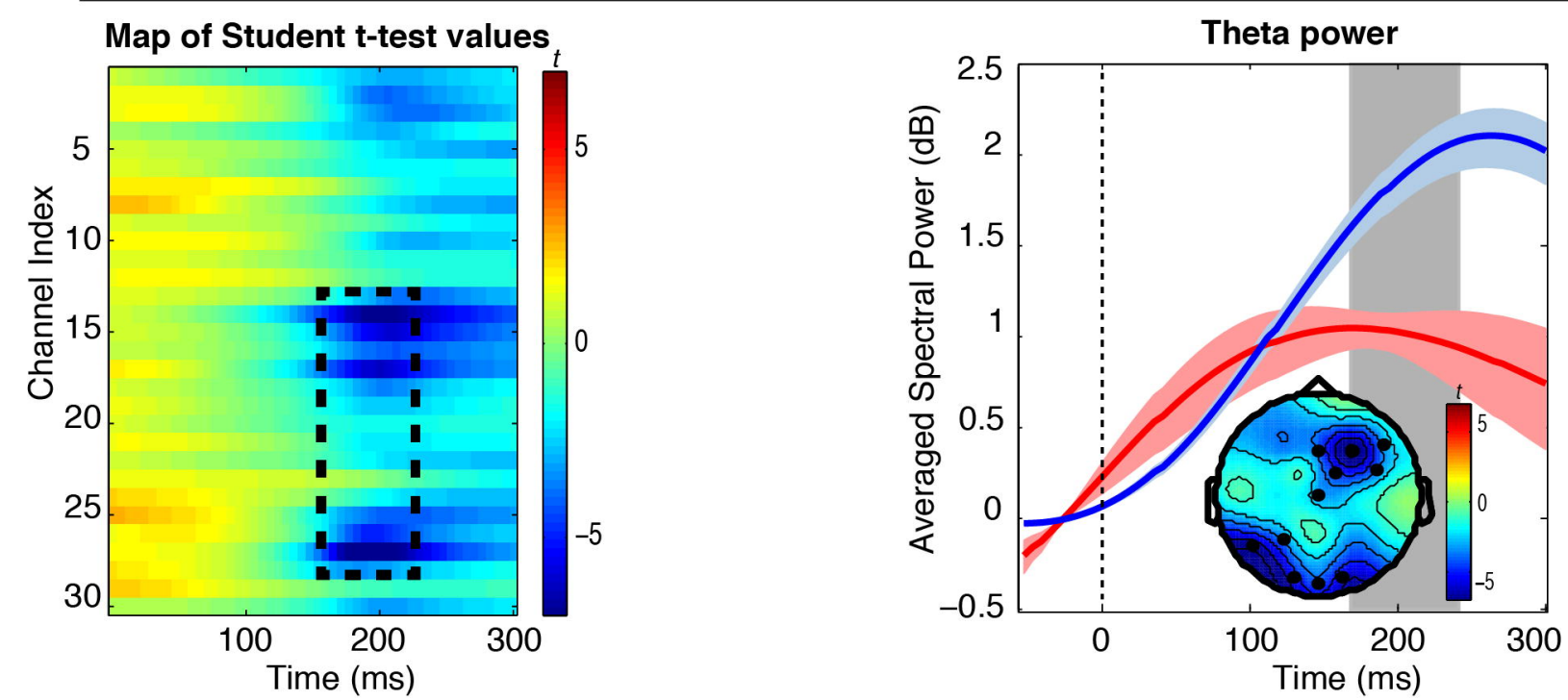
Table 1. Mean and 95\% confidence intervals of descriptive exercise-intensity parameters and behavioural performance for the moderate-to-high intensity and low intensity sessions.

\begin{tabular}{|c|c|c|c|c|}
\hline & \multicolumn{2}{|c|}{$\begin{array}{c}\text { Moderate-to-high intensity } \\
\left(80 \% \mathrm{VO}_{2 \max }\right)\end{array}$} & \multicolumn{2}{|c|}{$\begin{array}{l}\text { Light intensity } \\
\left(30 \% \mathrm{VO}_{2 \max }\right)\end{array}$} \\
\hline \multicolumn{5}{|c|}{ Exercise period parameters } \\
\hline Mean power load (W) & \multicolumn{2}{|c|}{$233.4 \pm 242.8$} & \multicolumn{2}{|c|}{$87.5 \pm 90.8$} \\
\hline $\begin{array}{l}\text { Mean relative power } \\
\text { load }(\mathrm{W} / \mathrm{kg})\end{array}$ & \multicolumn{2}{|c|}{$3.0 \pm 3.2$} & \multicolumn{2}{|c|}{$1.1 \pm 1.2$} \\
\hline \multicolumn{5}{|c|}{ Behavioural performance } \\
\hline & $\mathrm{ACC}(\%)$ & RT (ms) & $\operatorname{ACC}(\%)$ & RT (ms) \\
\hline Standard & $.99 \pm 1.0$ & --- & $1.0 \pm 1.0$ & --- \\
\hline Target 1 & $.90 \pm .9$ & $315.7 \pm 352.3$ & $.88 \pm .9$ & $320.6 \pm 363.8$ \\
\hline Target 2 & $.98 \pm 1.0$ & $234.7 \pm 256.5$ & $.97 \pm 1.0$ & $236.9 \pm 260.2$ \\
\hline
\end{tabular}

$\mathrm{W}=$ watios; $\mathrm{kg}=$ kilograms; $\mathrm{ACC}=$ accuracy; $\mathrm{RT}=$ reaction time 